\title{
Differential Depression at Excitatory and Inhibitory Synapses in Visual Cortex
}

\author{
Juan A. Varela, Sen Song, Gina G. Turrigiano, and Sacha B. Nelson \\ Department of Biology and Center for Complex Systems, Brandeis University, Waltham, Massachusetts 02254
}

The function of cortical circuits depends critically on the balance between excitation and inhibition. This balance reflects not only the relative numbers of excitatory and inhibitory synapses but also their relative strengths. Recent studies of excitatory synapses in visual and somatosensory cortices have emphasized that synaptic strength is not a fixed quantity but is a dynamic variable that reflects recent presynaptic activity. Here, we compare the dynamics of synaptic transmission at excitatory and inhibitory synapses onto visual cortical pyramidal neurons. We find that inhibitory synapses show less overall depression than excitatory synapses and that the kinetics of recovery from depression also differ between the two classes of synapse. When excitatory and inhibitory synapses are stimulated concurrently, this differential depression produces a timeand frequency-dependent shift in the reversal potential of the composite postsynaptic current. These results indicate that the balance between excitation and inhibition can change dynamically as a function of activity.

Key words: depression; temporal integration; temporal filtering; contrast adaptation; presynaptic; neocortex; nonlinearity; EPSC; IPSC; GABA
Cortical circuits are highly recurrent and are therefore intrinsically unstable. Even small reductions in the strength of cortical inhibition can result in epileptiform bursts (Chagnac-Amitai and Connors, 1989) and can disrupt the specificity of responses to sensory stimuli (Sillito, 1975; Nelson, 1991). Conversely, drugs that augment inhibition or reduce excitation can greatly diminish spontaneous and sensory evoked cortical activity (Dykes et al., 1984). The balance between excitation and inhibition is a key determinant of network behavior in simulations of cortical circuits (Somers et al., 1995; Tsodyks and Sejnowski, 1995; van Vreeswijk and Sompolinsky, 1996) and has a profound influence on long-term plasticity in the cortex (Kirkwood and Bear, 1994; Hensch et al., 1998).

During repetitive activation, excitatory synapses in the neocortex exhibit prominent frequency-dependent depression (Thomson et al., 1993; Markram and Tsodyks, 1996; Abbott et al., 1997; Tsodyks and Markram, 1997; Varela et al., 1997). Frequencydependent and paired-pulse depression (PPD) have also been described at inhibitory synapses in neocortex and hippocampus (Ben-Ari et al., 1979; McCarren and Alger, 1985; Deisz and Prince, 1989; Thompson and Gähwiler, 1989; Davies and Collingridge, 1993; Metherate and Ashe, 1994; Thomson et al., 1996; Reyes et al., 1998). Depression of excitatory and inhibitory synapses should produce opposite effects on cortical activity; depression of excitatory synapses will reduce recurrent excitation, whereas depression of inhibition will increase recurrent excitation. This suggests that the gain with which afferent signals are amplified or suppressed by cortical circuits will depend critically

\footnotetext{
Received Jan. 15, 1999; revised March 18, 1999; accepted March 22, 1999.

This work was supported by National Science Foundation Grant IBN 9511094, the Sloan Foundation, National Institutes of Health Grant EY11115, and the W. M. Keck Foundation. S.S. was supported by a Howard Hughes Medical Institute predoctoral fellowship. We thank Larry Abbott for helpful discussions and Chris Hempel for comments on this manuscript.

Correspondence should be addressed to Sacha Nelson, Department of Biology, Mail Stop 008, Brandeis University, Waltham, MA 02254.

Copyright (C) 1999 Society for Neuroscience 0270-6474/99/194293-12\$05.00/0
}

on the relative magnitudes and kinetics of depression at excitatory and inhibitory synapses. If depression at these synapses is closely matched, the balance between excitation and inhibition, and hence the overall gain of the circuit, will be relatively constant. On the other hand, if depression differs at these synapses, then the gain of the circuit may shift during repetitive stimulation. If depression is more prominent at inhibitory than excitatory synapses, the gain will increase with increasing activity, whereas if depression is more prominent at excitatory than inhibitory synapses, the gain will decrease.

Despite the importance of this issue, short-term plasticity of excitatory and inhibitory synapses onto pyramidal neurons have rarely been studied concurrently and have not been quantitatively compared. Hence, little is known about their relative impact on cortical activity. Recently, Galaretta and Hestrin (1998) have shown that, during very prolonged stimulation $(>200$ presynaptic action potentials), excitatory synapses show greater depression than inhibitory synapses. Here, we show that differential depression of excitatory and inhibitory synapses also occurs over a more rapid time scale ( $<10$ presynaptic action potentials). Pharmacologically isolated IPSCs exhibited less depression than EPSCs over a wide range of frequencies and after as few as 2-5 stimuli. As a consequence, when both are activated concurrently, there is a time- and frequency-dependent shift in the balance between excitation and inhibition to favor inhibition. This may permit cortical circuits to amplify afferent signals (Douglas et al., 1995; Somers et al., 1995), with a gain that is transiently quite high but that shifts over time to lower values, thus avoiding instability.

\section{MATERIALS AND METHODS}

Coronal slices containing primary visual cortex were obtained from Long-Evans rats, aged postnatal days 13-19, as described previously (Varela et al., 1997). Animals were deeply anesthetized with ketamine $(100 \mathrm{mg} / \mathrm{kg})$ and acepromazine $(10 \mathrm{mg} / \mathrm{kg})$ or pentobarbital $(35 \mathrm{mg} / \mathrm{kg})$ and decapitated, and their brains were quickly removed and placed in chilled $\left(5^{\circ} \mathrm{C}\right)$ artificial CSF (ACSF). Slices of $400 \mu \mathrm{m}$ thickness were cut on a vibratome. During recording, slices were transilluminated to permit 
visualization of the location of primary visual cortex and the boundaries between layers 2/3 and 4 (Dominici et al., 1995).

Slices were maintained at room temperature on semipermeable membranes (Falcon 3090) covered by a thin layer of ACSF continuously oxygenated with $95 \% \mathrm{O}_{2}-5 \% \mathrm{CO}_{2}$. They were transferred one at a time to a submerged chamber mounted on a fixed-stage upright microscope (Optiphot UD; Nikon, Tokyo, Japan) and slowly warmed to $32-35^{\circ} \mathrm{C}$. Slices were equilibrated for $1-2 \mathrm{hr}$ before recording and remained viable for up to $16 \mathrm{hr}$. Slices were perfused with warmed oxygenated ACSF at a rate of $2-3 \mathrm{ml} / \mathrm{min}$. For obtaining visually guided whole-cell recordings, slices were illuminated obliquely through an infrared filter and viewed with standard optics using a $40 \times$ long-working distance water immersion objective. The resulting image was displayed on a video monitor using a CCD camera. Pyramidal neurons were identified on the basis of their pyramidally shaped somata and single apical dendrites.

Solutions. ACSF contained (in mM): $126 \mathrm{NaCl}, 3 \mathrm{KCl}, 1.25 \mathrm{NaH}_{2} \mathrm{PO}_{4}$, 10 dextrose, $20 \mathrm{NaHCO}_{3}, 2 \mathrm{MgSO}_{4}$, and $2.0 \mathrm{CaCl}_{2}, \mathrm{pH} 7.4$ when saturated with $95 \% \mathrm{O}_{2}-5 \% \mathrm{CO}_{2}$ (osmolarity, 310-315 mOsm). Pipettes were pulled from $1.0 \mathrm{~mm}$ outer diameter thin-walled capillary tubing (Warner Instruments, Hamden, CT) on a Flaming-Brown horizontal puller (Sutter Instruments, Novato, CA). Pipettes were filled with (in $\mathrm{mM}$ ): $125 \mathrm{~K}$-methylsulfonate, $10 \mathrm{KCl}, 3 \mathrm{~K}_{2} \mathrm{ATP}, 1 \mathrm{Na}_{2} \mathrm{GTP}$, and 2 $\mathrm{MgSO}_{4}$. The solution also contained $10 \mathrm{~mm}$ HEPES and $1 \mathrm{~mm}$ BAPTA to buffer intracellular $\mathrm{pH}$ and calcium. The quartenary lidocaine derivative QX-314 (10 mM; Bromide salt, $n=15$ neurons; chloride salt, $n=$ 49 neurons) was also included to block sodium action potentials and postsynaptic $\mathrm{GABA}_{\mathrm{B}}$ receptors. Osmolarity of internal solution was 290-300 mOsm, pH 7.3-7.4.

Electrophysiological techniques. Whole-cell recording pipette resistances were 2-4 $\mathrm{M} \Omega$ in the bath. Voltage-clamp recordings were performed using an Axopatch 1D (Axon Instruments, Foster City, CA). Seal resistances were 2-8 G $\Omega$. Series resistance and input resistance were measured before each synaptic stimulus, and recordings were rejected if these or the resting membrane potential changed by $>20 \%$. Steady-state voltage errors resulting from series resistance $(10.0 \pm 2.0 \mathrm{M} \Omega)$ were not compensated for recordings of small $(<200 \mathrm{pA})$ currents at membrane potentials close to rest. We estimate that these errors were $<1 \mathrm{mV}$. For determining reversal potentials of synaptic currents, the steady-state voltage errors were corrected post hoc to obtain more accurate measurements. For experiments in which large synaptic currents were evoked (see Fig. 8), voltage errors could be appreciable, and so we also repeated these experiments in five neurons using $65-85 \%$ series resistance compensation. (Before compensation, series resistance in these neurons was 7-9 $\mathrm{M} \Omega$.) The reversal potentials measured using series resistance compensation and post hoc holding voltage correction $(n=9)$ differed by $<2$ $\mathrm{mV}$, which was not statistically significant $(t$ test; $p=0.71)$. Voltages were not corrected for liquid junction potentials (which were measured and found to be $<3 \mathrm{mV}$ ). Postsynaptic currents (PSCs) were judged to be monosynaptic if they occurred with short $(1.5-4.0 \mathrm{msec})$ and constant (jitter $<1 \mathrm{msec}$ ) latency that did not change with small changes in stimulus strength. To isolate AMPA-mediated EPSCs, low-amplitude electrical stimuli were applied through a patch pipette, and the position was adjusted to one in which additional inhibitory components were not present after depolarization (to $0 \mathrm{mV}$ ). In some cases, low doses (2.5-5.0 $\mu \mathrm{M}$ ) of bicuculline were added to the bath to partially block $\mathrm{GABA}_{\mathrm{A}^{-}}$ mediated IPSPs. To isolate $\mathrm{GABA}_{\mathrm{A}}$-mediated IPSCs, electrical stimuli were applied while AMPA receptors were blocked with $10 \mu \mathrm{M}$ CNQX. In previous experiments performed on similar slices and using similar stimulation protocols, we found that fiber excitability as assessed by the amplitude of a nonsynaptic antidromic and fiber volley response was constant, even at high frequencies (Varela et al., 1997). In all experiments, NMDA receptors were blocked with $50 \mu \mathrm{M}$ APV or with $2 \mu \mathrm{M}$ (+)-5-methyl-10,11-dihydro-5H-dibenzo [a,d] cyclohepten-5,10-imine maleate (MK-801), and action potentials and postsynaptic $\mathrm{GABA}_{\mathrm{B}}$ receptors were blocked intracellularly. Except where stated, IPSCs were measured as inward currents at $-90 \mathrm{mV}$ to reduce changes in chloride reversal potential, which can occur during large outward $\mathrm{GABA}_{\mathrm{A}}$ currents (Thompson and Gähwiler, 1989). EPSCs were measured at $-70 \mathrm{mV}$.

Analysis. Response magnitudes were measured as peak amplitudes within a $1 \mathrm{msec}$ window. For IPSCs, contamination of response peaks with polysynaptic responses was not of concern because the presence of CNQX and APV blocked polysynaptic propagation. For EPSCs, we also measured, in a subset of cells, the slope during the initial $1 \mathrm{msec}$ of the response. Initial slopes were very strongly correlated with peak responses
(Pearson correlation coefficient; $r>0.98 ; n=6$ cells), suggesting that polysynaptic contamination was minimal. For most measurements, the baseline amplitude immediately preceding the stimulus artifact was subtracted. For the measurements of Figures $5 B$ and 8 , we wished to measure the summated synaptic current and therefore used a baseline period immediately preceding the entire stimulus train.

Amplitudes of responses to constant frequency trains were normalized to the initial response and fit with exponential functions of the form:

$$
1-A e^{-N / B}
$$

where $N$ is the number of stimuli in the train, $B$ is the number of stimuli producing an $e$-fold decline in response amplitude, and $1-A$ is the steady-state amplitude. The amount of depression during the train was measured from the steady-state of the fitted exponential or from the average normalized amplitude of the last four responses in the train. Both methods yielded similar results. For most measurements, we did not extrapolate the preceding current and subtract the extrapolated residual. However, for several cells, we performed this extrapolation (see Fig. $2 D$, asterisks) and found virtually no difference, even for the fastest frequencies tested $(100 \mathrm{~Hz})$.

Amplitudes of responses to Poisson-distributed trains were fit with one-component (Eq. 2) and two-component (Eq. 3) models of synaptic depression as described previously (Varela et al., 1997):

$$
\begin{gathered}
A=A_{0} D, \\
A=A_{0} D_{1} D_{2} .
\end{gathered}
$$

Dynamic variables representing depression $(D)$ were constrained to be $\leq 1$ and depended on the stimulus pattern in the following way. After each stimulus in the train, $D$ was multiplied by a constant factor $(d)$ representing the amount of depression per presynaptic action potential:

$$
D \rightarrow D d
$$

Between stimuli, $D$ recovered exponentially back toward 1 with firstorder kinetics and time constant $\tau_{\mathrm{D}}$ :

$$
\tau_{D} d D / d t=1-D
$$

For two-component fits, $D_{1}$ and $D_{2}$ had different constant factors $\left(d_{1}, d_{2}\right)$ and time constants $\left(\tau_{\mathrm{D} 1}, \tau_{\mathrm{D} 2}\right)$.

For Poisson-distributed trains, we also calculated a depression index (DI), which was simply the average amplitude of responses in the train normalized to the amplitude of the first response in the train. Fits were evaluated by measuring the root mean square of the deviations of measured points from predicted points.

\section{RESULTS}

To compare the dynamics of synaptic transmission at excitatory and inhibitory synapses in primary visual cortical slices, we evoked monosynaptic EPSCs and IPSCs in visually identified layer $2 / 3$ pyramidal neurons with random and constant frequency trains. Because we have previously characterized short-term synaptic dynamics for excitatory synapses in layer 2/3 (Varela et al., 1997), we focus here primarily on short-term plasticity of IPSCs and on additional recordings of EPSCs, which allowed direct comparison of excitation and inhibition over a wider frequency range $(0.1-100 \mathrm{~Hz})$.

Synaptic responses were evoked by extracellular stimuli through a patch pipette placed $60-100 \mu \mathrm{m}$ away. Monosynaptic GABAergic IPSPs were recorded in the presence of CNQX (10 $\mu \mathrm{M})$ and APV $(50 \mu \mathrm{M})$ or MK-801 $(2 \mu \mathrm{M})$ to block AMPAmediated and NMDA-mediated synaptic transmission (Fig. 1). Postsynaptic $\mathrm{GABA}_{\mathrm{B}}$ responses were blocked by including the quartenary lidocaine derivative QX-314 (10 mM) in the pipette solution (Nathan et al., 1990) so that the remaining currents were likely to be almost exclusively a result of activation of $\mathrm{GABA}_{\mathrm{A}}$ receptors. These currents were abolished by addition of bicuculline (10 $\mu \mathrm{M} ; n=5$; data not shown). For neurons recorded with internal solution containing the chloride salt of QX-314, the 
A

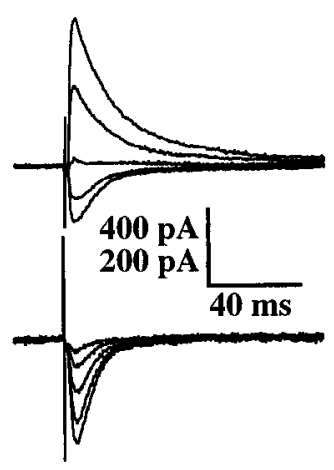

B

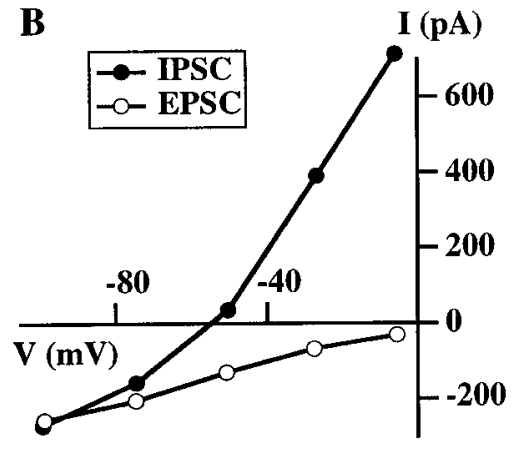

Figure 1. Isolated EPSCs and IPSCs recorded in layer $2 / 3$ during local extracellular stimulation. $A$, Monosynaptic IPSCs (top traces) and EPSCs (bottom traces) recorded during whole-cell voltage clamp of two different layer $2 / 3$ pyramidal neurons. Recordings were made during blockade of NMDA receptors and $\mathrm{GABA}_{\mathrm{B}}$ receptors. IPSCs were recorded in the presence of CNQX to block AMPA receptors. Intrinsic whole-cell currents have been subtracted. Each trace is the average of three (IPSC) or six (EPSC) repetitions. $B, I-V$ curve for the peak synaptic currents shown in $A$.

reversal potential of the IPSC was $-49.4 \pm 2.2 \mathrm{mV}$ (SEM; $n=$ $21)$, close to the chloride equilibrium potential calculated from the Nernst equation $(-48.6 \mathrm{mV})$. Evoked currents exhibited prominent outward rectification. The decay was biexponential, with time constants of $20.7 \pm 4.1$ and $120.0 \pm 5.4 \mathrm{msec}(n=16)$ at $-90 \mathrm{mV}$.

EPSCs were recorded under similar conditions, except that CNQX was omitted from the bath, and the stimulus current and position were adjusted to yield activation of excitatory inputs without measurable activation of inhibition (Fig. 1). As expected, EPSCs had a more rapid decay than IPSCs (single exponential, $6.9 \pm 0.9 \mathrm{msec} ; n=16)$ and reversed near $0 \mathrm{mV}(-1.3 \pm 3.4 \mathrm{mV}$; $n=16)$.

\section{Differential depression of isolated IPSCs and EPSCs during constant frequency stimulation}

To characterize short-term plasticity at inhibitory synapses in visual cortex, we measured responses to constant frequency trains over a range of frequencies from 0.1 to $100 \mathrm{~Hz}$. The amplitudes of IPSCs were stable at stimulation frequencies of $0.1 \mathrm{~Hz}$ and below. At higher frequencies, IPSC amplitudes decayed exponentially toward a steady-state amplitude that diminished with increasing frequency. Figure 2 illustrates IPSCs recorded from one neuron during eight different frequencies of stimulation. Figure $2 A-C$, left, shows average responses ( $n \geq 7$ repetitions) to trains at 0.1 , 5 , and $50 \mathrm{~Hz}$. Figure 2D, right, shows the measured IPSC amplitudes for trains at $0.1,0.5,10$, and $100 \mathrm{~Hz}$. The decline in amplitudes for $0.5 \mathrm{~Hz}$ and above were reasonably well fit by single exponentials. The steady-state amplitudes varied with frequency, but the number of stimuli required for the amplitude to fall 1/e of the way toward steady-state was approximately similar across frequencies $(2.52,2.92$, and 3.05 for $0.5,10$, and $100 \mathrm{~Hz})$. This meant that, regardless of frequency, the IPSC amplitude reached $\sim 95 \%$ of its steady-state value within the first 10 stimuli. The temporal evolution of depression was similar in other neurons tested $(n=20)$, except that depression at low frequencies $(0.5-5$ $\mathrm{Hz}$ ) often had a more rapid onset, with the majority of the depression occurring between the first and second stimuli (see below).
Figure $2 E$ illustrates the dependence of the steady-state amplitude on stimulus frequency for this neuron. The curve has two clearly separate portions: an initial portion over which steadystate amplitude declined rapidly with increasing frequency for low frequencies, and a second portion over which steady-state amplitude declined more slowly with increasing frequency for higher frequencies. For this neuron, the transition between the two portions of the curve occurred somewhere between 1 and $5 \mathrm{~Hz}$. The relationship between depression at the end of the train and frequency was well fit by the sum of two exponentials with critical frequencies (i.e., the frequency change over which there is an $e$-fold change in amplitude) of 1.1 and $63.3 \mathrm{~Hz}$, respectively. Other neurons tested $(n=20)$ showed a similar pattern of frequency dependence, although the kinetics and overall amplitude of the depression varied from neuron to neuron (Figs. 3-5).

We and others have recently found that excitatory synaptic inputs to neocortical pyramidal neurons exhibit strong depression that reduces the response amplitude to a level inversely proportional to the stimulating frequency (Abbott et al., 1997; Tsodyks and Markram, 1997). In contrast, our initial recordings of isolated IPSCs revealed depression that was weaker and that varied less steeply with frequency. To confirm this, we compared depression of IPSCs recorded from each of 20 neurons with that of isolated EPSCs recorded in 16 additional pyramidal neurons in the absence of CNQX. Two example recordings are shown in Figure 3. EPSCs recorded in one neuron held at $-70 \mathrm{mV}$ are compared with IPSCs recorded in another neuron held at $-90 \mathrm{mV}$. In both cases, the neurons were stimulated with a train of 15 stimuli at 20 $\mathrm{Hz}$. The EPSCs exhibited very strong depression to $8.0 \pm 2.5 \%$ of their initial amplitude by the end of the train, whereas the depression of the IPSCs was more modest, reaching only of $37.8 \pm 3.0 \%$ of their initial amplitude.

The time course of depression of EPSCs and IPSCs averaged across all neurons tested are compared in Figure 4 for four different frequencies. For each neuron, the amplitudes of synaptic responses were normalized to that of the first response in the train and then averaged across neurons. The temporal evolution of depression varied with frequency. At $0.5 \mathrm{~Hz}$, there was PPD between the first and second stimuli in the train for both EPSCs and IPSCs but little subsequent depression. At $5 \mathrm{~Hz}$, PPD was comparable for EPSCs and IPSCs, but subsequently, depression built up more rapidly for EPSCs than for IPSCs. This trend became more prominent at higher frequencies, so that at 20 and $50 \mathrm{~Hz}$ the curves were well separated. Two-way ANOVA (PSC type, stimulus number within the train) performed separately for PSCs at each frequency, revealed a significant dependence $(p<$ 0.05 ) of PSC amplitude on stimulus number for all frequencies 0.2 $\mathrm{Hz}$ and above and a significant difference $(p<0.05)$ between EPSCs and IPSCs for $1 \mathrm{~Hz}$ and above.

Depending on the release probability, excitatory synapses in neocortex may exhibit initial facilitation before depression (Markram and Tsodyks, 1996; Thomson, 1997; Tsodyks and Markram, 1997; Varela et al., 1997). At higher frequencies ( $\geq 20$ $\mathrm{Hz}$ ), 7 of 25 of the neurons tested exhibited either facilitation or a reduced rate of depression of the PSCs during the first several stimuli within the train. Neuron-to-neuron variation in the degree of initial depression versus facilitation appears to account for the greater variance early in the train than toward the end of the train. It is important to point out, however, that even PSCs showing strong initial facilitation were strongly depressed after the first 6-10 stimuli.

The majority of previous studies of short-term plasticity of 


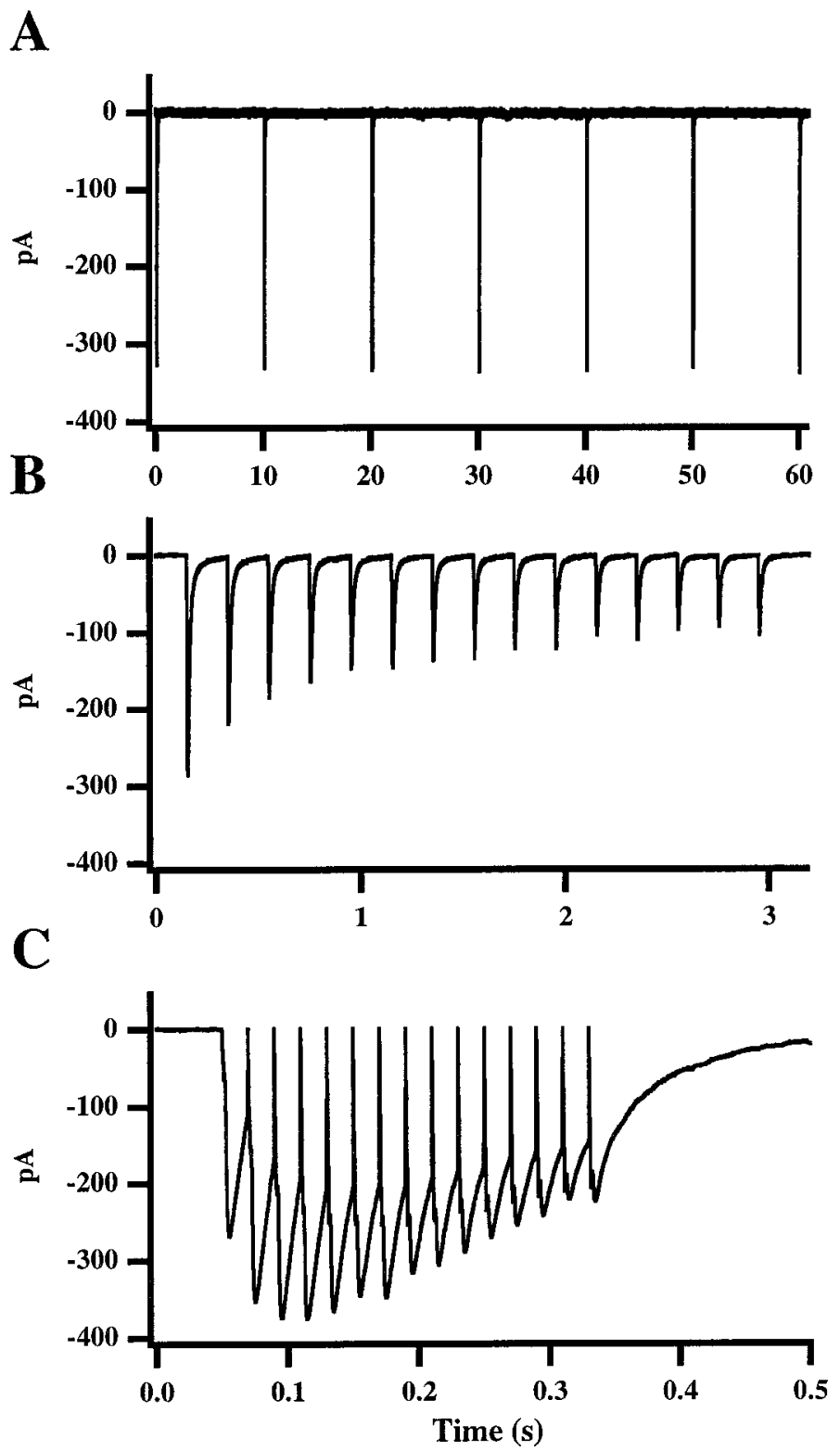

D

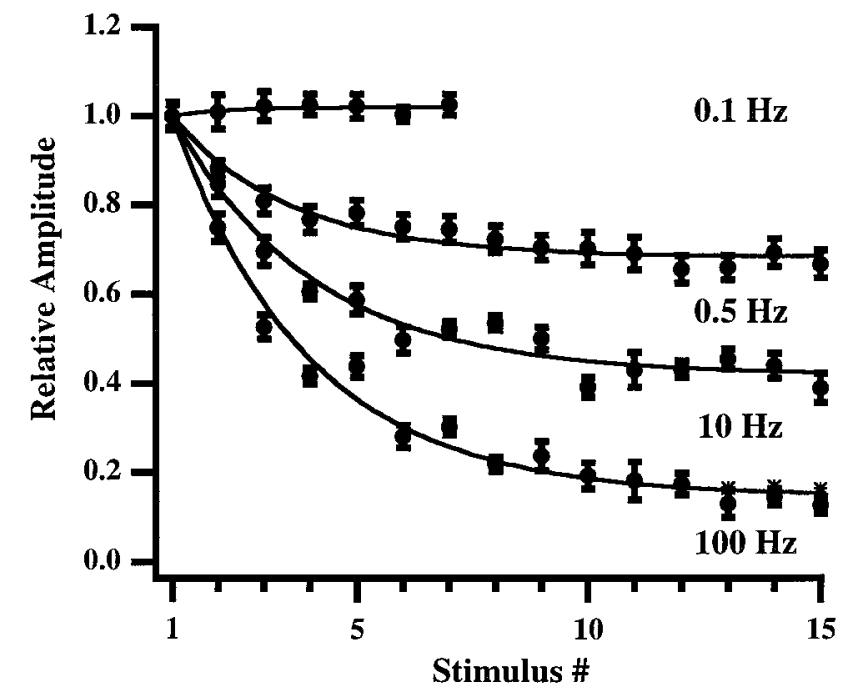

$\mathbf{E}$

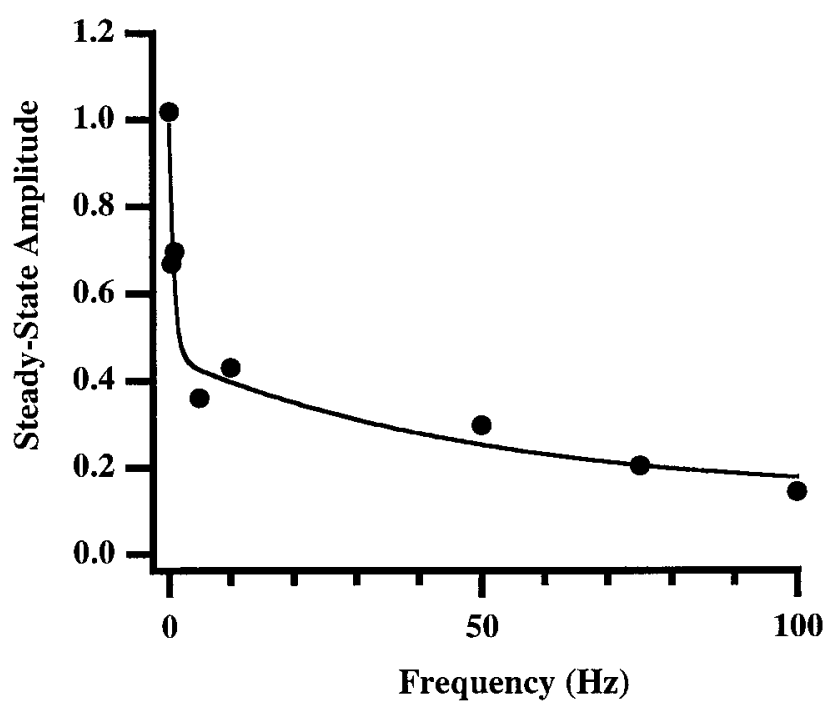

Figure 2. Frequency-dependent depression of IPSCs during constant frequency trains. IPSCs were evoked in one pyramidal neuron by constant frequency trains at eight different frequencies ranging from 0.1 to $100 \mathrm{~Hz}$. Each train consisted of $15 \mathrm{stimuli}$, except for trains at $0.1 \mathrm{~Hz}$, which consisted of only seven stimuli $(n=15$ repetitions for $0.1,1,10$, and $100 \mathrm{~Hz} ; n=7$ repetitions for $0.5,5,50$, and $75 \mathrm{~Hz})$. $V_{\text {hold }},-90 \mathrm{mV}$. $A-C$, Average IPSCs for $0.1(A), 5(B)$, and $50(C) \mathrm{Hz}$. $D$, Response amplitudes from data in $A$ and $B$ and from two other frequencies $(10 \mathrm{and} 100 \mathrm{~Hz})$. Additional frequencies are omitted for clarity. Amplitudes are normalized to the initial response. Responses were measured relative to a baseline immediately preceding each stimulus. Asterisks indicate relative amplitudes of three last responses at $100 \mathrm{~Hz}$ in which preceding responses were fit with exponentials and extrapolated to permit more accurate baseline measurements (see Materials and Methods). Error bars indicate SEM; lines are linear $(0.1 \mathrm{~Hz})$ or single exponential fits $(0.5-100 \mathrm{~Hz}) . E$, Steady-state depression (average relative amplitude of the last 4 responses in each train) for each of the eight frequencies tested. Steady-state depression values were fitted with the sum of two exponentials.

central synapses have focused on paired-pulse facilitation and PPD. We observed that paired-pulse interactions often did not provide an accurate estimate of steady-state behavior. To compare paired-pulse and steady-state behavior quantitatively, we measured the average paired-pulse interaction by computing the ratio of the second response in the train to the first as a function of the stimulus frequency (the inverse of the paired-pulse interval) (Fig. 5A). As previously reported for hippocampal IPSCs (Davies et al., 1990), PPD of IPSCs was a nonmonotonic function of stimulus frequency. PPD first increased with frequency, reaching a maximum at between 2 and $5 \mathrm{~Hz}$ (200-500 msec interval), and then decreased, reaching a minimum at $20 \mathrm{~Hz}$. At higher frequencies, PPD increased more slowly. PPD of EPSCs was similar, although there was less depression of EPSCs at 10 and 20 $\mathrm{Hz}$, presumably because of concurrent facilitation. A two-way ANOVA (frequency, PSC type) revealed a significant dependence on frequency $\left(p=10^{-4}\right)$ but no significant overall difference between EPSCs and IPSCs $(p=0.252)$.

In contrast to paired-pulse effects, steady-state depression increased monotonically across the measured range of frequencies. We measured the steady-state depression at each frequency by computing the ratio of the average of the last four responses in 
A

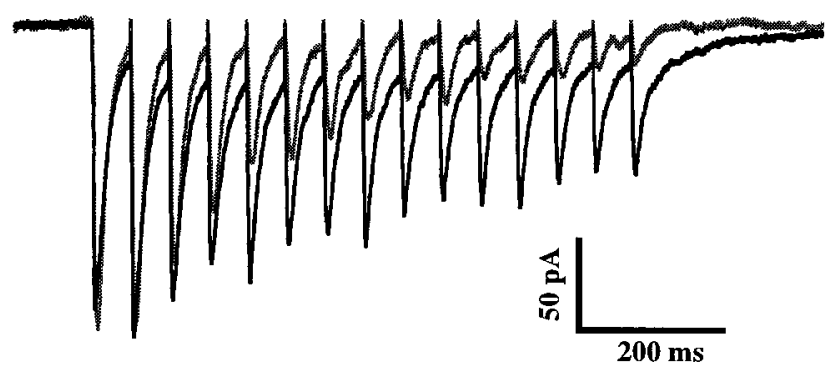

B

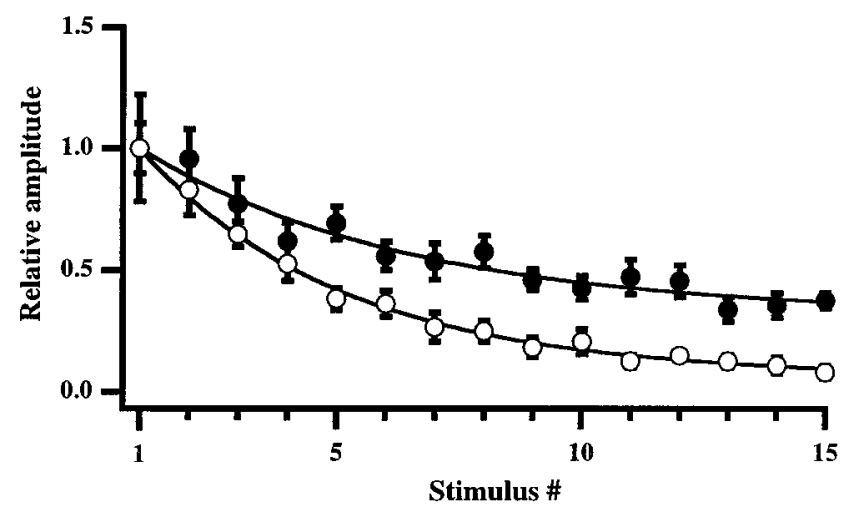

Figure 3. Differential depression of IPSCs and EPSCs at $20 \mathrm{~Hz}$. Data are from two different visually identified pyramidal neurons. $A$, Superimposed traces are average IPSCs (black line; $n=7$ repetitions) recorded in one cell in the presence of CNQX and APV at $-90 \mathrm{mV}$ and average EPSCs (gray line; $n=7$ repetitions) recorded in another cell in the presence of APV at $-70 \mathrm{mV}$. $B$, Amplitudes of the data in $A$ normalized to the initial response. Error bars indicate SEM. Fits are single exponential functions.

the train to the initial response. Figure $5 B$ shows the frequency dependence of the steady-state depression for EPSCs and IPSCs averaged across neurons. The smooth curves are double exponential fits. The curves diverge above $5 \mathrm{~Hz}$ and are widely separated over the remainder of the frequency range tested. The difference in the steady-state depression of EPSCs and IPSCs was highly significant ( $p=0.008$; two-factor ANOVA; frequency, PSC type). We also measured the steady-state depression by fitting the average responses obtained at each frequency with a single exponential decay (Fig. 2D). The results obtained with this method were nearly identical (data not shown).

In addition to the fact that EPSCs and IPSCs exhibit different rates of synaptic depression, they also differed in their decay kinetics and hence showed different degrees of temporal summation. To determine how depression and summation interact to influence the balance of excitatory and inhibitory current, we measured the summed synaptic current from the same responses plotted in Figure $5 B$. In this case (Fig. $5 C$ ), the baseline measurement subtracted from each response was made immediately before the first stimulus in the train rather than immediately preceding each response. As expected, the effects of summation only became significant at higher frequencies and, at any given fre-
A

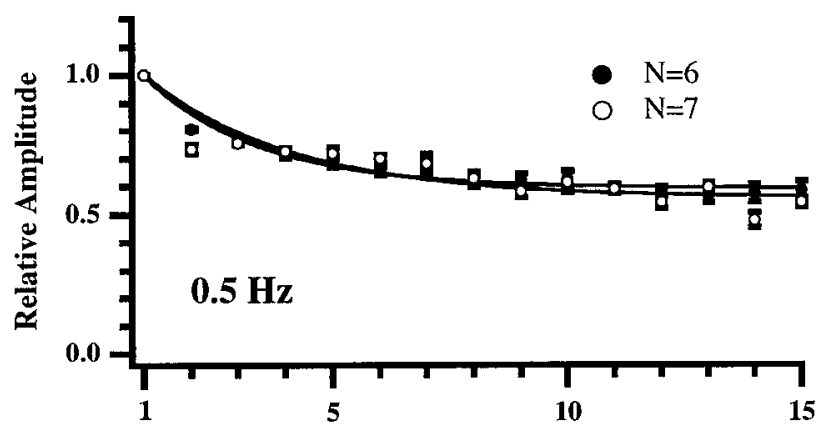

B
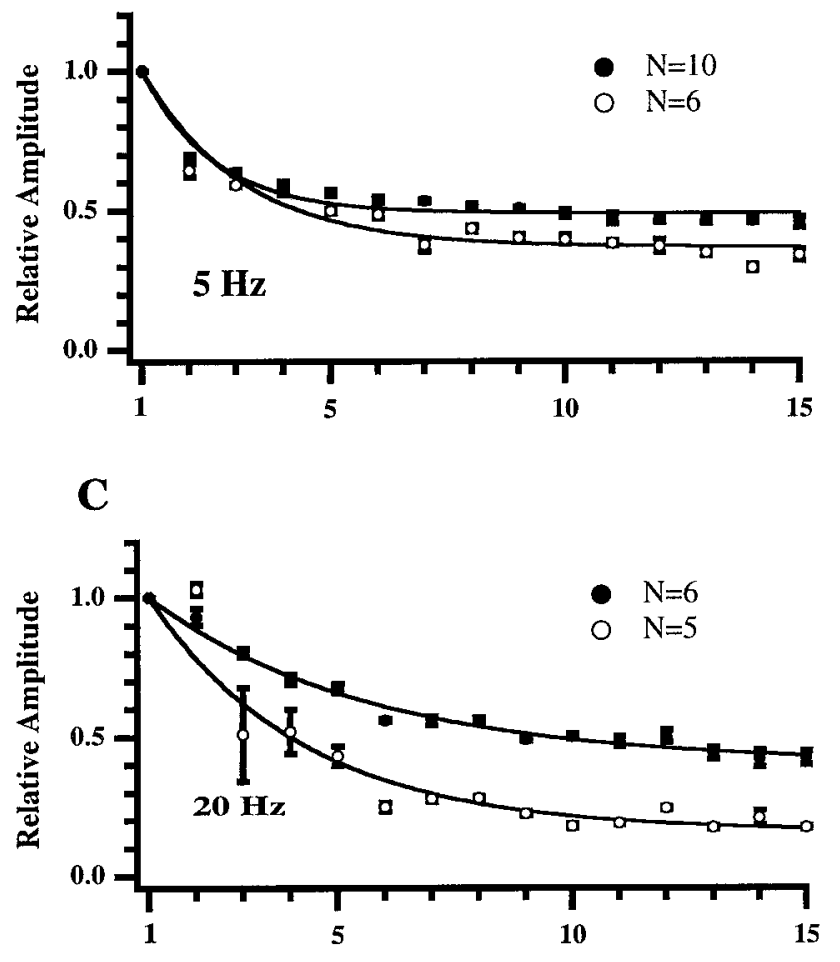

D

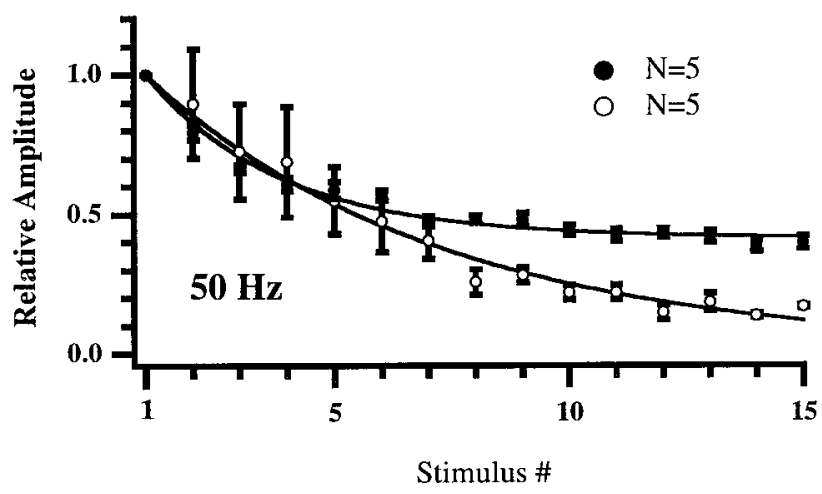

Figure 4. Differential synaptic depression of IPSCs and EPSCs averaged across cells. IPSCs ( filled circles) and EPSCs (open circles) evoked by constant frequency trains (15 stimuli) at the frequencies indicated. Average data for each cell were normalized to the first response and then averaged across cells. Error bars indicate SEM; $n$ indicates number of cells tested. Fits are single exponential functions. 
A

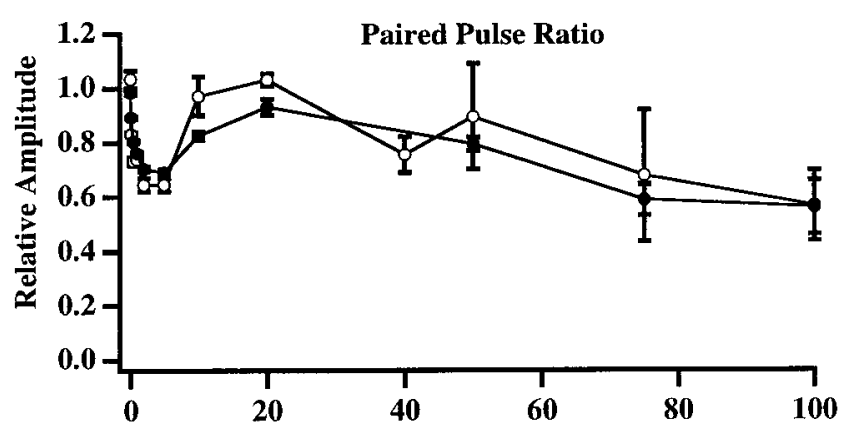

B

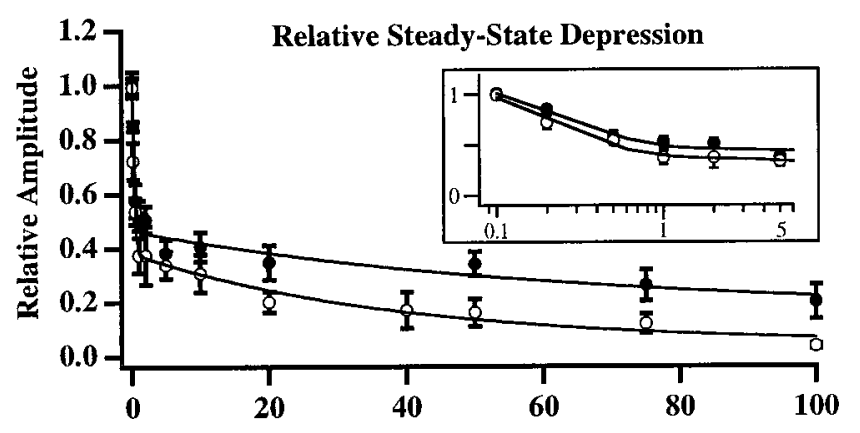

C

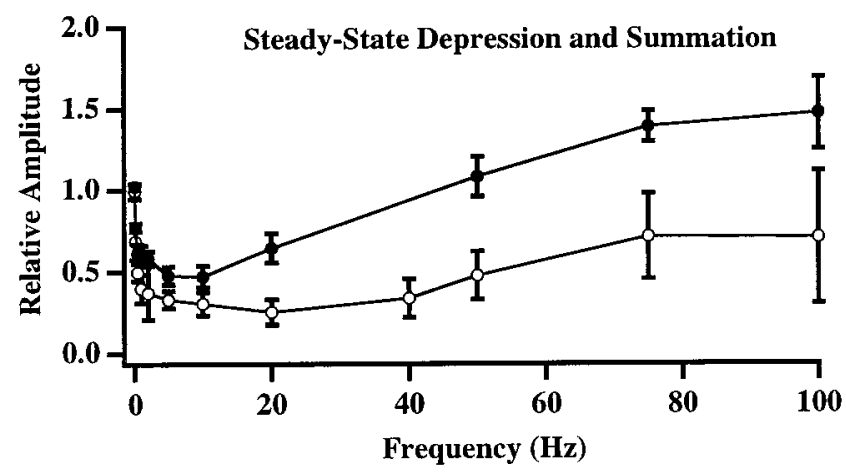

Figure 5. Frequency dependence of depression and summation for EPSCs and IPSCs. A-C, Filled circles indicate IPSCs, and open circles indicate EPSCs. $A$, Paired-pulse depression, measured as the ratio of the amplitude of the second stimulus to that of the first. Note that pairedpulse depression is similar for IPSCs and EPSCs and is a poor predictor of steady-state behavior above $5-10 \mathrm{~Hz}$. B, Steady-state depression of incremental current. The ratio of the average amplitudes of the last four responses in each train to the initial response are plotted for each frequency tested. As in Figures 2-4, PSC amplitudes are measured as differences between baseline immediately preceding the stimulus and the peak current after the stimulus. Data are fit with the sum of two exponentials. $C$, Steady-state depression of summated synaptic current. Amplitudes of summated currents are measured as differences between peak current and the baseline preceding the entire train, so amplitude reflects both depression and summation of successive responses. Note that, because of greater summation of IPSCs than EPSCs, the difference between the two curves is accentuated above $20 \mathrm{~Hz}$.

quency, were more pronounced for IPSCs than for EPSCs. This led to a widening of the difference between the curves for EPSCs and IPSCs for frequencies between 20 and $100 \mathrm{~Hz}(p=8 \times$ $10^{-9}$; two-factor ANOVA; frequency, PSC type).

\section{Differential depression of IPSCs and EPSCs during random stimulation}

We have shown previously that an efficient method of characterizing short-term plasticity at cortical synapses is to stimulate with trains of stimuli that contain a random mixture of frequencies (Varela et al., 1997). Figure 6 illustrates the IPSCs evoked in a layer $2 / 3$ pyramidal neuron by Poisson-distributed stimulus trains applied locally during blockade of excitatory transmission. IPSCs exhibited modest depression that was typically most prominent between the first two stimuli in the train and that recovered completely during the $40 \mathrm{sec}$ interval between repetitions of the train.

To characterize these responses quantitatively, we fit the data with one- and two-component models of short-term synaptic depression (Eqs. 2, 3; see also Varela et al., 1997). An example of one such fit is shown in Fig. $6 D$. The root mean square error was $7.1 \%$, indicating an accurate fit. For this neuron, a single component of depression (parameters shown in Fig. $6 C$ ) provided an excellent fit to the data, which could not be improved by the addition of a second component of depression (i.e., the best fit with the two-component model was one in which the second depression constant did not contribute; $d_{2}, 1$ ). This was true for 14 of 15 neurons studied. Fits to IPSCs from the remaining neuron showed a modest improvement (10.7 vs $15.5 \%)$ when using the two-component model.

Figure 7 shows a comparison of the two-component fit parameters obtained for IPSCs with those obtained previously for EPSCs. In general, the trains of IPSCs were well described by a single component of depression that reduced response amplitude by $\sim 6 \%$ per presynaptic action potential $\left(d_{1}, 0.94 \pm 0.07\right)$ and that recovered with a time constant of $\sim 1.9 \mathrm{sec}$. These results are in contrast to those obtained previously for EPSCs and field potentials (Varela et al., 1997), which were generally much better described by two components of depression: one faster and stronger than the depression of IPSCs $\left(d_{1}, 0.78 \pm 0.06 ; \tau_{1}\right.$, $634 \pm 96 \mathrm{msec})$ and the second weaker but much slower $\left(d_{2}\right.$, $\left.0.97 \pm 0.008 ; \tau_{2}, 9.3 \pm 1.8 \mathrm{sec}\right)$. The average depression per stimulus (DI) was $0.80 \pm 0.02$ for IPSCs, indicating less than half of the average depression observed previously for EPSCs (DI, $0.53 \pm 0.30$ ).

We assessed the ability of the one- and two-component models to accurately predict the constant frequency data in Figures 4 and 5. Fits were in general satisfactory for the range of frequencies between 1 and $50 \mathrm{~Hz}$, but the same set of parameters could not accurately predict responses at very low $(<1 \mathrm{~Hz})$ or very high $(100$ $\mathrm{Hz}$ ) frequencies. Specifically, at low frequencies, more depression than predicted was observed, whereas at high frequencies, less depression than predicted was observed. Accurate fits to the entire range could easily be obtained if recovery time constants were assumed to be slower at low frequencies than at high frequencies. These discrepancies suggest that our initial formulation in which recovery from depression occurs at a fixed rate may need to be modified to predict responses when mean rates fluctuate over several orders of magnitude. Several recent studies suggest that a more complete and accurate description of shortterm plasticity at central synapses requires a model in which the rates of recovery from depression are not fixed but vary with activity (Dittman and Regehr, 1998; Klingauf et al., 1998; Stevens and Wesseling, 1998; Wang and Kaczmarek, 1998). 

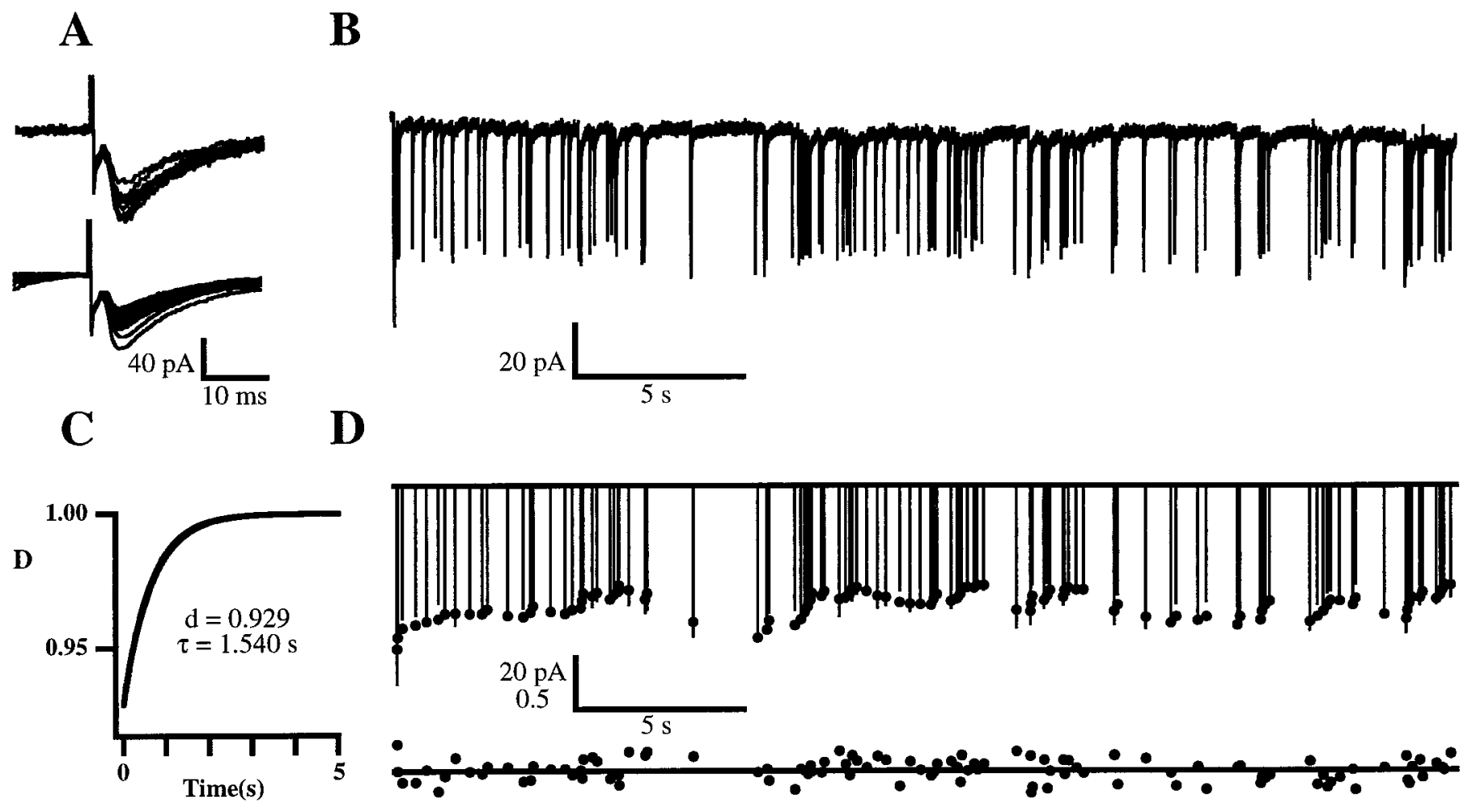

Figure 6. Measured and predicted amplitudes of IPSCs evoked by random stimulus trains. Monosynaptic IPSCs evoked by a Poisson-distributed stimulus train (mean rate, $4 \mathrm{~Hz}$; duration, $30 \mathrm{sec}$ ) recorded from a pyramidal neuron held at $-90 \mathrm{mV}$. A, Top, Individual responses to the first stimulus of the train ( $n=9$ repetitions). Bottom, Average responses (across repetitions) to each of the 112 stimuli in the train. $B$, Averaged responses to the entire train shown at a reduced time scale. $C$, Parameters of the best fit of a single-component model of synaptic depression (see Materials and Methods). The curve illustrates the amplitude and time course of the recovery of depression after a single stimulus. D, Top, Measured (lines) and predicted (dots) amplitude of the IPSCs. Bottom, The fraction of each measured response amplitude by which the model prediction differed from the data.

\section{Shifting balance between IPSCs and EPSCs evoked concurrently}

A limitation of the comparison between depression of EPSCs and IPSCs in Figures 3-8 is that it relies on population comparisons, because it was not feasible to hold recordings long enough to sequentially isolate and adequately study EPSCs and IPSCs in a single neuron. In addition, it is possible that silencing EPSCs may remove other modulatory influences that modify the short-term plasticity of IPSCs. As a means of circumventing these limitations, we studied the dynamics of EPSCs and IPSCs evoked concurrently. To do this, we measured the reversal potential of compound PSCs consisting of a mixture of $\mathrm{GABA}_{\mathrm{A}}$ receptormediated inhibition and AMPA receptor-mediated excitation. A change in the relative contributions of IPSCs and EPSCs to the compound PSC should result in a shift in that reversal potential. These responses were evoked using trains of large amplitude stimuli in the presence of APV and internal QX-314 to block NMDA and $\mathrm{GABA}_{\mathrm{B}}$ receptors. For each neuron, we evoked a train of compound PSCs at 40 or $50 \mathrm{~Hz}$ at a range of holding potentials and then measured the reversal potential of the response as a function of stimulus number within the train. Figure $8 A$ illustrates the records obtained in one such experiment. Each stimulus in the train evoked a mixture of inward and outward synaptic currents. The reversal potential of the composite PSC evoked by the first stimulus was $-36 \mathrm{mV}$ (Fig. $8 C$, right-most curve). During the course of the train, the reversal potential of the composite PSC shifted to $-47 \mathrm{mV}$ (Fig. $8 C$, left-most curve). Previous studies have found that activity can cause a shift in the chloride equilibrium potential in cortical and hippocampal neurons (Thompson et al., 1988). To determine whether or not this contributed to the shift in the reversal potential of the combined PSC, we applied CNQX to isolate the monosynaptic IPSC evoked by the same stimuli in the same neurons. In the presence of CNQX, the inward current was greatly reduced (Fig. $8 B$ ). The remaining synaptic current reversed at $-49 \mathrm{mV}$, close to the reversal potential expected for the isolated $\mathrm{GABA}_{\mathrm{A}}$-mediated IPSC. The reversal potential did not change during the train (Fig. $8 D$ ), indicating that there was little shift in the chloride equilibrium potential, which may have accounted for the shift observed under control conditions. Similar results were obtained for 13 neurons in which we measured the shift in the reversal potential of the composite PSC and for seven of these neurons in which we subsequently measured the reversal potential of the isolated IPSC (Fig. 8E). In contrast to the dramatic shift in reversal potential seen for responses to high-frequency trains, the reversal potential for responses to $5 \mathrm{~Hz}$ trains was constant ( $n=3$ neurons; data not shown).

Because the large stimuli used activated a complex cortical circuit, it is likely that multiple factors contributed to the observed shift in reversal potential. For example, it is possible that repeated stimulation may have selectively depressed polysynaptic input from excitatory neurons or may have selectively potentiated polysynaptic input from inhibitory neurons (see Discussion). In general, it was more difficult to cleanly differentiate monosynaptic and polysynaptic responses when large, complex synaptic responses were evoked. It is unlikely that these circuit-level effects 
A

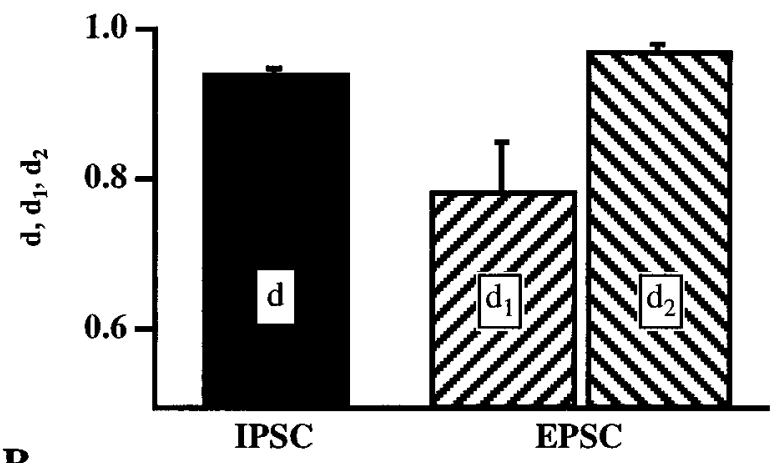

B
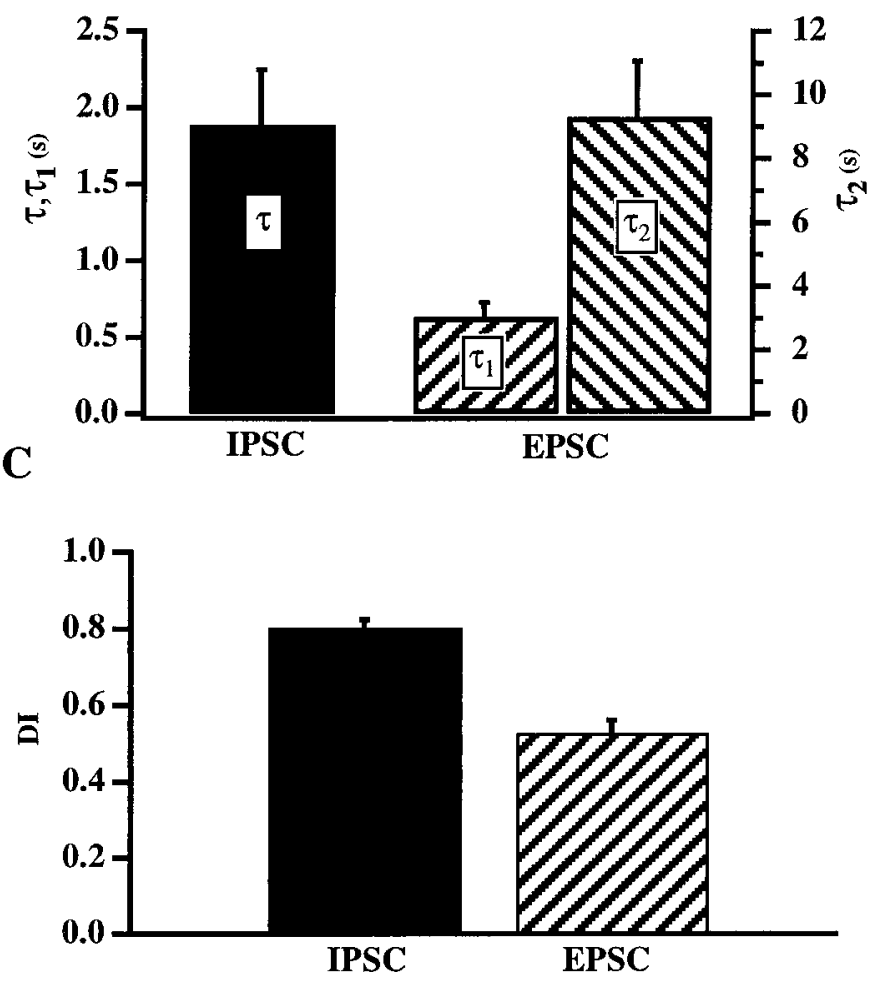

Figure 7. Comparison of depression of IPSCs and EPSCs during random stimulation. One-component and two-component models of depression (see Materials and Methods) were fit to IPSCs obtained during random stimulation at a mean frequency of $4 \mathrm{~Hz}$ (solid bars; $n=15$ cells) as shown in Figure 6. Fit parameters shown are for the one-component model. Fit parameters (two-component model) for random stimulation of EPSCs at the same mean frequency (hatched bars; $n=9$ cells) are replotted from Varela et al. (1997) for comparison. A, Magnitudes of depression per stimulus. $B$, Time constants of recovery from depression. $\tau$ (IPSC) and $\tau_{1}$ (EPSC) are plotted on the left axis, and $\tau_{2}$ (EPSC) is plotted on the right axis because of its longer duration. $C$, Depression indices, average depression during random stimulus trains measured as the mean amplitude of each response in the train normalized to the amplitude of the first response.

were the sole cause of the shift, however, because the shift was prominent, even when measuring the earliest $1 \mathrm{msec}$ of responses which occurred at latencies of only $1-3 \mathrm{msec}$. (The reversal potential of the composite PSC shifted from $-33.6 \pm 3.1$ to $-45.4 \pm 3.1 \mathrm{mV} ; n=9$.) This implies that a major determinant of the observed shift is differential depression of monosynaptic excitatory and inhibitory input.
The rate of depression of isolated EPSCs and IPSCs was similar at low frequencies and differed most dramatically at frequencies above $20 \mathrm{~Hz}$ (Figs. 4, 5). This implies that, at lower frequencies, the amount of recurrent excitation onto pyramidal neurons and hence the effective gain of the circuit, is higher. For a given level of afferent input, this higher gain should increase firing rates. As firing rates increase, however, the balance between excitation and inhibition will shift to favor inhibition and will drive firing rates back down. In principle, the behavior of such a network should depend strongly on the initial ratio of excitation to inhibition. The importance of this initial ratio is illustrated schematically in Figure 9. The figure illustrates the relative steady-state amplitudes of EPSCs and IPSCs (replotted from Fig. 5) for a fixed level of inhibition and for various relative levels of excitation. If initial inhibitory strength equals or exceeds excitatory strength (Fig. 9, curve marked $1 X$ ), increasing frequency only widens the difference in the levels of excitation and inhibition, thereby keeping the balance tipped toward inhibition. If, on the other hand, the initial level of excitation is higher (Fig. 9, curves marked $1.5 X, 2 X$, and $2.5 X)$, the balance between excitation and inhibition flips from one favoring excitation at low frequencies to one favoring inhibition at higher frequencies. The point at which this flip occurs is the point at which the EPSC and IPSC curves cross. At higher initial excitatory strengths, the crossover point occurs at higher firing rates.

\section{DISCUSSION}

The central finding of this study is that, during repeated stimulation, inhibitory synapses on to layer $2 / 3$ pyramidal neurons exhibit significantly less synaptic depression than do excitatory synapses. This difference emerges rapidly, often within the first three to five stimuli in a train, and depends on frequency, becoming more prominent at higher frequencies. A crucial determinant of pyramidal neuron firing is the balance between its excitatory and inhibitory synaptic input. The present results indicate that this balance is not fixed but is subject to rapid modification by activity. The direction of this modification favors stability; increased activity shifts the balance in favor of inhibition. If instead, increased activity had shifted the balance in favor of excitation, repeated stimulation would be expected to evoke larger and larger responses, eventually leading to epileptiform discharge. Indeed, such a destabilizing shift in the balance between excitation and inhibition has been hypothesized to underlie epilepsy in some brain regions, such as the hippocampus and other limbic structures (Ben-Ari et al., 1979; McCarren and Alger, 1985).

Galaretta and Hestrin (1998) recently demonstrated a slowly accumulating form of depression that was much more pronounced and recovered more slowly at excitatory synapses than at inhibitory synapses. They concluded, as we do here, that this should shift the balance between cortical excitation and inhibition to favor inhibition. A key difference between these studies is that Galaretta and Hestrin focused on a much longer time scale of activity (hundreds of presynaptic action potentials vs $\leq 15$ here). Results obtained in this study using random frequency trains support the idea that slow depression is more pronounced at excitatory than at inhibitory synapses. Previously, we found that depression at excitatory synapses could best be described by a two-component model containing a rapid component of depression accumulating over the course of a few presynaptic action potentials and recovering in a few hundred milliseconds and a second component that accumulated and recovered more slowly (onset, 1-3\% per action potential; $\tau, 5-20 \mathrm{sec}$ ) (Varela et al., 


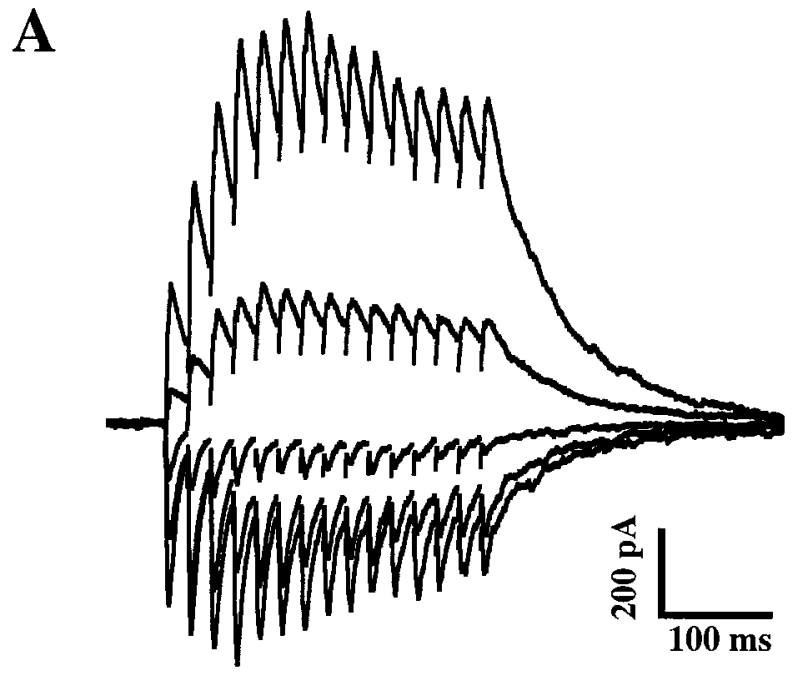

B
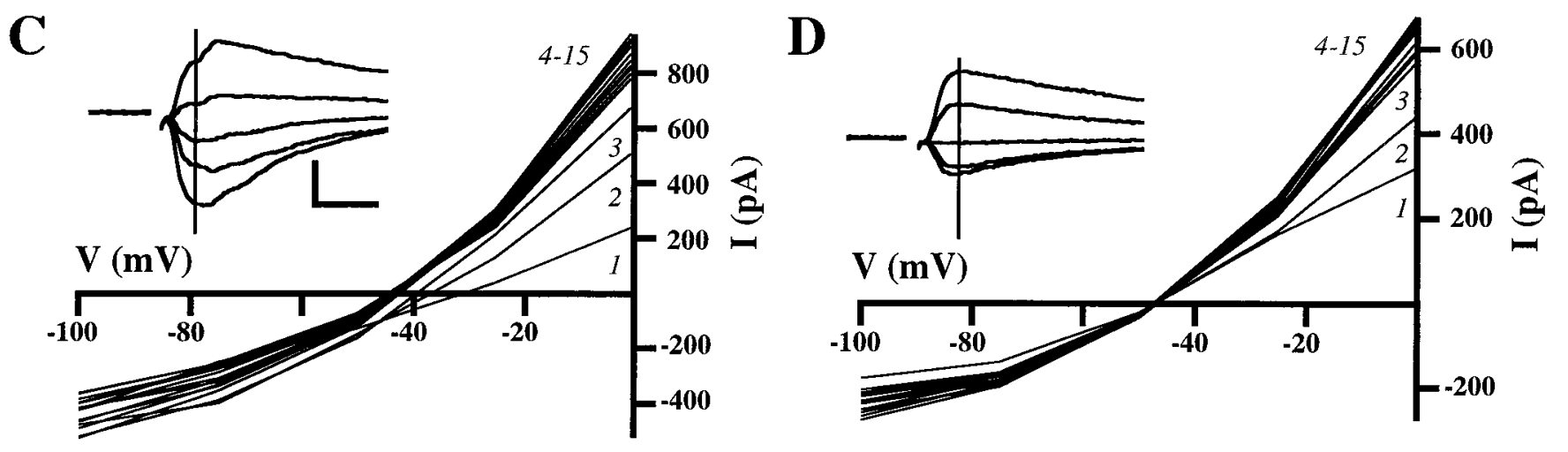

$\mathbf{E}$

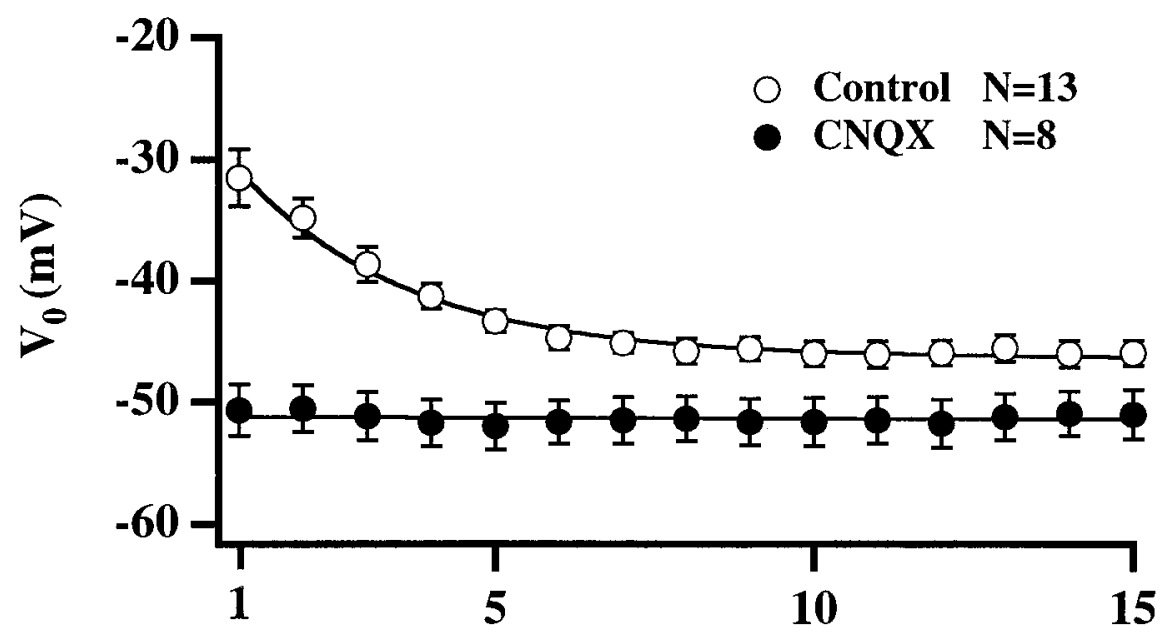

\section{Stimulus \#}

Figure 8. The reversal potential of composite PSCs shifts during repeated stimulation. $A$, Composite PSCs evoked in a layer $2 / 3$ pyramidal neuron held at five different potentials $(-100,-75,-50,-25$, and $-1.0 \mathrm{mV})$ by a high-amplitude stimulus train. Stimulus train consists of $15 \mathrm{stimuli}$ at $50 \mathrm{~Hz}$. Intrinsic whole-cell currents evoked by the voltage stops have been subtracted. Each trace is the average of five repetitions. Calibration: 200 pA, 50 msec. $B$, Monosynaptic IPSCs evoked in the same cell by an identical stimulus train after addition of CNQX. Calibration is the same as in $A$. $C, D, I-V$ relationships for the synaptic currents shown in $A$ and $B$. Each line is the $I-V$ curve for a single stimulus in the train. (Figure legend continues) 


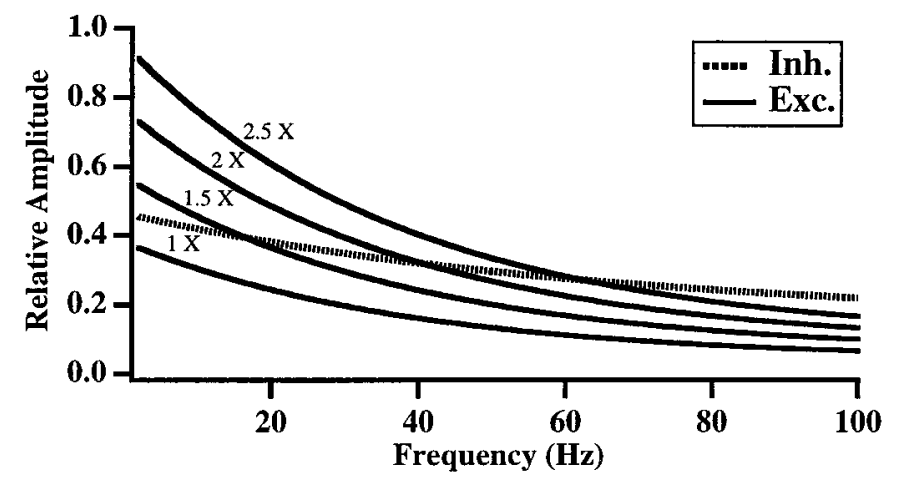

Figure 9. Frequency-dependent changes in the balance between excitation and inhibition. Curves fit to steady-state depression data in Figure $5 B$ are replotted in the range of $1-100 \mathrm{~Hz}$. Original curves for inhibition (dashed line) and excitation (solid line, $1 X$ ) do not cross, because they are normalized to 1 at $0 \mathrm{~Hz}$ (i.e., the amplitude of a single stimulus). Solid curves marked $1.5 \mathrm{X}, 2 \mathrm{X}$, and $2.5 \mathrm{X}$ indicate curves for excitation if initial EPSC amplitudes exceeds IPSC amplitudes by a factor of $1.5,2$, or 2.5 . These curves are generated by multiplying the $1 \mathrm{X}$ curve by the appropriate factor. For these cases, the curves for excitation and inhibition cross at progressively higher frequencies.

1997). In contrast, we found in these experiments that IPSCs evoked by random trains could be well described by a single component of depression with an intermediate rate of onset $(6 \%)$ and recovery $(2 \mathrm{sec})$. Galaretta and Hestrin did not observe the differences in more rapid depression found here. The reason for this discrepancy is not apparent, but it may reflect differences in the way in which rapid depression was quantified (primarily as averages over the first 50 stimuli within a train) or in the population of synapses studied (primarily layer 5 somatosensory cortex).

Differential depression is only one of several important factors that are likely to act synergistically to produce an activitydependent shift in the balance between cortical excitation and inhibition. For example, several recent studies have found that excitatory input from pyramidal neurons on to some classes of inhibitory interneurons facilitate (Thomson, 1997; Markram et al., 1998; Reyes et al., 1998). Like differential depression, this would tend to boost inhibition onto pyramidal neurons relative to excitation. Differences in intrinsic firing properties are also likely to play an important role. Pyramidal neuronal firing accommodates, whereas most (although not all) interneurons do not accommodate, and are potentially able to fire at higher rates (McCormick et al., 1985). Finally, although it has been widely observed previously that EPSCs in neocortex and hippocampus decay more rapidly than IPSCs, the functional impact of this fact on the frequency-dependent buildup of excitation and inhibition has rarely been assessed quantitatively. The present results indicate that differential summation and differential depression act together to shift the balance between excitation and inhibition. It is likely that filtering introduced by somatic whole-cell voltage clamp has led us to underestimate the speed of the fastest PSCs. If so, this should have a much greater effect on the faster and more distally located EPSCs than on IPSCs and so may have led us to underestimate the degree of differential summation.

It is important to point out that the frequency-dependent depression examined here is, at least for frequencies above $2 \mathrm{~Hz}$, primarily distinct from paired-pulse depression. It was not generally possible to predict the steady-state behavior (Fig. $5 B$ ) from the paired-pulse behavior (Fig. 5A). This suggests that, to understand how synaptic dynamics influence the dynamics of cortical activity, it is not sufficient to use pairs of stimuli. Mechanistically, paired-pulse effects appear to reflect a mixture of facilitation (Fleidervish and Gutnick, 1995), presynaptic inhibition (Deisz and Prince 1989; Davies et al., 1990), and other presynaptic factors (Wilcox and Dichter, 1994). The result is a complex, nonmonotonic dependence on frequency. Other than ruling out a significant contribution from changes in chloride reversal potential, we have not examined the mechanisms responsible for the depression at inhibitory synapses observed during stimulus trains. By analogy with previous work at excitatory synapses, however, it seems likely that depression reflects depletion of a readily releasable pool of transmitter or a cumulative inactivation of some component of the release machinery. Activation of presynaptic receptors for adenosine, GABA, or glutamate may have influenced the short-term plasticity observed. We have shown previously, for example, that short-term plasticity at excitatory synapses is strongly influenced by activation of $\mathrm{GABA}_{\mathrm{B}}$ receptors and adenosine receptors (Varela et al., 1997). Some modulators, such as adenosine, differentially affect excitatory and inhibitory synapses (Varela et al., 1995) and so could alter the dynamic balance between cortical excitation and inhibition. It is unlikely, however, that differential depression was caused by differences in the modulators released under the different conditions used for studying isolated excitatory and inhibitory synapses, because the effect was also observed when both sets of synapses were stimulated concurrently.

An important issue not addressed by the qualitative model of cortical dynamics presented here is the impact of potential differences between the dynamics of synapses made by different classes of inhibitory neurons. Such classes have been defined on the basis of firing pattern, molecular phenotype, morphology, and axonal targets (Kawaguchi and Kubota, 1993). The use of extracellular stimulation in the present study did not allow identification of differences that may exist between dynamics of IPSCs arising from different classes of interneurons. However, several recent studies involving paired recordings from interneurons and pyramidal neurons have failed to reveal significant differences in the short-term plasticity exhibited by synapses onto pyramidal neurons from different classes of inhibitory interneurons (Thomson et al., 1996; Tamás et al., 1997; Reyes et al., 1998; TasrczyHonoch et al., 1998). Additional paired-recording studies may reveal important differences not yet documented, and this may necessitate a more refined model of the dynamic balance between excitation and inhibition.

A key aspect of our approach was to measure the dynamics of the net postsynaptic current evoked when many synaptic inputs to pyramidal neurons were activated concurrently. The behavior we

\section{$\leftarrow$}

Vertical lines in insets indicate the latency at which currents were measured. Amplitudes were measured relative to baseline preceding train and so reflect both depression and summation. Calibration: $200 \mathrm{pA}, 5 \mathrm{msec}$. E. Average reversal potentials for composite PSCs (open circles) and monosynaptic IPSCs ( filled circles). For each cell, the reversal potential was determined for each response in the train, from the interpolated zero crossing of $I-V$ plots like those shown in $C$ and $D$. Plots show mean \pm SEM across all cells tested with single exponential (control) and linear (CNQX) fits. 
observed, a time- and frequency-dependent shift in the reversal potential of the compound PSC, was that predicted on the basis of measurements of isolated EPSCs and IPSCs. This implies that additional factors engaged when many synapses are stimulated simultaneously are either less important than, or act in concert with, differential depression. Studies of the dynamic behaviors of populations of interacting cortical neurons stimulated synchronously or asynchronously may offer an arena in which to more rigorously relate biophysical properties of single cells and synapses to information processing in cortical circuits.

Another important issue requiring further study is the developmental time course of differential depression. We found previously that the slow form of depression at excitatory synapses declines with age (Varela et al., 1997). Reyes and Sakmann (1998) have also reported recently a decline in depression with age, although their study focused primarily on paired-pulse effects. Considerations of stability suggest that decreased depression at excitatory synapses should be accompanied by decreased depression at inhibitory synapses. Otherwise, high-frequency trains should regularly evoke epileptiform events in visual cortex, something not typically observed in slices from older animals.

The results presented here have focused exclusively on the fast

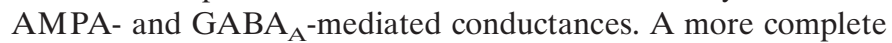
analysis of the balance between excitation and inhibition will also need to consider the effects of the weaker, but much longer lasting, $\mathrm{GABA}_{\mathrm{B}}$ and NMDA conductances. Presynaptic and postsynaptic forms of depression have been described previously for these conductances (Otis et al., 1993; Tong et al., 1995), but it will be important in future work to assess how these contribute to the overall balance between excitation and inhibition.

The shifting balance between excitation and inhibition demonstrated here has important implications for the way that activity evolves over time in recurrent circuits. Circuit-level models of the cortex have generally not included synaptic dynamics and have therefore dealt primarily with the regime in which the circuit behaves as an amplifier (Douglas et al., 1995; Somers et al., 1995), the regime in which it behaves as an attenuator (Kyriazi et al., 1996; Troyer et al., 1998), or the nearly balanced state (Shadlen and Newsome, 1994; Tsodyks and Sejnowski 1995; van Vreeswijk and Sompolinsky, 1996). Instead, it may be more realistic to consider these different activity regimes as arising from the same underlying circuit dynamics. Circuits operating at high gain are sensitive and can activate rapidly, but they saturate easily (Troyer and Miller, 1997). Dynamic adjustment of gain with activity may offer the optimal compromise between the opposing constraints of speed and sensitivity on the one hand and dynamic range and stability on the other. Recently, Borg-Graham et al. (1998) and Moore and Nelson (1998) have observed dramatic changes in the apparent reversal potential of sensory-evoked synaptic currents in cortical neurons in vivo. These changes are more complex than those evoked by trains of electrical stimuli and include both initial shifts in the balance between excitation and inhibition like those described here (Borg-Graham et al., 1998, their Fig. 4a; Moore and Nelson, 1998, their Fig. 7C), as well as subsequent shifts back from inhibition toward rebound excitation later in the response. These results underscore the dynamic nature of the balance between excitation and inhibition in shaping the sensory responses of cortical neurons.

Differential depression may also contribute to temporal patterns of cortical activation during repeated sensory stimulation. In visual cortex, prolonged stimulation with effective stimuli evokes activity that diminishes or "adapts" with time. Recently,
Carandini and Ferster (1997) have reported that contrast adaptation is accompanied by a tonic hyperpolarization of the membrane potential of visual cortical neurons. Although it remains possible that changes in intrinsic conductances contribute to this phenomenon (Sanchez-Vives et al., 1998), a shift in the balance between synaptic activity arising from spontaneously active excitatory and inhibitory afferents could also contribute if prolonged visual stimulation led to greater depression of excitatory afferents (Chance et al., 1998; Galaretta and Hestrin, 1998). Resolution of this issue will require further experiment and perhaps more detailed simulation of the expected effects of differing patterns of intrinsic and synaptic dynamics.

\section{REFERENCES}

Abbott LF, Sen K, Varela JA, Nelson SB (1997) Synaptic depression and cortical gain control. Science 275:220-222.

Ben-Ari Y, Krnjevic K, Reinhardt W (1979) Hippocampal seizures and failure of inhibition. Can J Physiol Pharmacol 57:1462-1466.

Borg-Graham LJ, Monier C, Frégnac Y (1998) Visual input evokes transient and strong shunting inhibition in visual cortical neurons. Nature 393:369-373.

Carandini M, Ferster D (1997) A tonic hyperpolarization underlying contrast adaptation in cat striate cortex. Science 276:949-952.

Chagnac-Amitai Y, Connors BW (1989) Horizontal spread of synchronized activity in neocortex and its control by GABA-mediated inhibition. J Neurophysiol 61:747-758.

Chance F, Nelson SB, Abbott LF (1998) Synaptic depression and the temporal response characteristics of V1 cells. J Neurosci 18:4785-4799.

Davies CH, Collingridge GL (1993) The physiological regulation of synaptic inhibition by $\mathrm{GABA}_{\mathrm{B}}$ autoreceptors in rat hippocampus. J Physiol (Lond) 472:245-265.

Davies CH, Davies SN, Collingridge GL (1990) Paired-pulse depression of monosynaptic GABA-mediated inhibitory postsynaptic responses in rat hippocampus. J Physiol (Lond) 424:513-531.

Deisz R, Prince D (1989) Frequency-dependent depression of inhibition in guinea-pig neocortex in vitro by GABAB receptor feedback on GABA release. J Physiol (Lond) 412:513-541.

Dittman JS, Regehr WG (1998) Calcium-dependence and recovery kinetics of presynaptic depression at the climbing fiber to Purkinje cell synapse. J Neurosci 18:6147-6162.

Dominici L, Harding GW, Burkhalter A (1995) Patterns of synaptic activity in forward and feedback pathways within rat visual cortex. J Neurophysiol 74:2649-2664.

Douglas RJ, Koch C, Mahowald M, Martin KAC, Suarez HH (1995) Recurrent excitation in neocortical circuits. Science 269:981-985.

Dykes RW, Landry P, Metherate R, Hicks TP (1984) Functional role of GABA in cat primary somatosensory cortex: shaping receptive fields of cortical neurons. J Neurophysiol 52:1066-1093.

Fleidervish IA, Gutnick MJ (1995) Paired-pulse facilitation of IPSCs in slices of immature and mature mouse somatosensory neocortex. J Neurophysiol 73:2591-2595.

Galaretta M, Hestrin S (1998) Frequency-dependent synaptic depression and the balance of excitation and inhibition in the neocortex. Nat Neurosci 1:587-594.

Hensch TK, Fagiolini M, Mataga N, Stryker MP, Baekkeskov S, Kash SF (1998) Local GABA circuit control of experience-dependent plasticity in developing visual cortex. Science 282:1504-1508.

Kawaguchi Y, Kubota Y (1993) Correlation of physiological subgroupings of nonpyramidal cells with parvalbumin- and calbindinD28kimmunoreactive neurons in layer $\mathrm{V}$ of rat frontal cortex. J Neurophysiol 70:387-396.

Kirkwood A, Bear MF (1994) Hebbian synapses in visual cortex. J Neurosci 14:1634-1645.

Klingauf J, Kavalali ET, Tsien RW (1998) Kinetics and regulation of fast endocytosis at hippocampal synapses. Nature 394:581-585.

Kyriazi HT, Carvell GE, Brumberg JC, Simons DJ (1996) Quantitative effects of GABA and bicuculline methiodide on receptive field properties of neurons in real and simulated whisker barrels. J Neurophysiol 75:547-560.

Markram H, Tsodyks MV (1996) Redistribution of synaptic efficacy between neocortical pyramidal neurons. Nature 382:807-810.

Markram H, Wang Y, Tsodyks M (1998) Differential signaling via the 
same axon of neocortical pyramidal neurons. Proc Natl Acad Sci USA 95:5323-5328.

McCarren M, Alger BE (1985) Use-dependent depression of IPSPs in rat hippocampal pyramidal cells in vitro. J Neurophysiol 53:557-571.

McCormick DA, Connors BW, Lighthall JW, Prince DA (1985) Comparative electrophysiology of pyramidal and sparsely spiny stellate neurons of the neocortex. J Neurophysiol 54:782-803.

Metherate R, Ashe JH (1994) Facilitation of an NMDA receptormediated EPSP by paired-pulse stimulation in rat neocortex via depression of GABAergic IPSPs. J Physiol (Lond) 481:331-348.

Moore CI, Nelson SB (1998) Spatio-temporal subthreshold receptive fields in the vibrissa representation of rat primary somatosensory cortex. J Neurophysiol 80:2882-2892.

Nathan T, Jensen MS, Lambert JDC (1990) The slow inhibitory postsynaptic potential in rat hippocampal CA1 neurones is blocked by intracellular injection of QX-314. Neurosci Lett 110:309-313.

Nelson SB (1991) Temporal interactions in the cat visual system. III. Pharmacological studies of cortical suppression suggest a presynaptic mechanism. J Neurosci 11:369-380.

Otis TM, De Koninck Y, Mody I (1993) Characterization of synaptically elicited $\mathrm{GABA}_{\mathrm{B}}$ responses using patch-clamp recordings in rat hippocampal slices. J Physiol (Lond) 463:391-407.

Reyes A, Sakmann B (1998) Layer specific and developmentally regulated short-term modification of unitary EPSPS in neocortical pyramidal neurons. Soc Neurosci Abstr 24:318.

Reyes A, Lujan R, Rozov A, Burnashev N, Somogyi P, Sakmann B (1998) Target-cell-specific facilitation and depression in neocortical circuits. Nat Neurosci 1:279-285.

Sanchez-Vives MV, McCormick DA, Nowak LG (1998) Is synaptic depression prevalent in vivo and does it contribute to contrast adaptation? Soc Neurosci Abstr 24:896.

Shadlen MN, Newsome WT (1994) Noise, neural codes and cortical organization. Curr Opin Neurobiol 4:569-579.

Sillito AM (1975) The contribution of inhibitory mechanisms to the receptive field properties of neurones in the striate cortex of the cat. J Physiol (Lond) 250:305-329.

Somers DC, Nelson SB, Sur M (1995) An emergent model of orientation selectivity in cat visual cortical simple cells. J Neurosci 15:5448-5465.

Stevens CF, Wesseling JF (1998) Activity-dependent modulation of the rate at which synaptic vesicles become available to undergo exocytosis. Neuron 21:415-424

Tamás G, Buhl EH, Somogyi P (1997) Fast IPSPs elicited via multiple synaptic release sites by different types of GABAergic neurone in the cat visual cortex. J Physiol (Lond) 500:715-738.

Tasrczy-Honoch K, Martin KAC, Jack JJB, Stratford KJ (1998) Synaptic interactions between smooth and spiny neurones in layer 4 of cat visual cortex in vitro. J Physiol (Lond) 508:351-363.
Thompson SM, Gähwiler BH (1989) Activity-dependent disinhibition. I. Repetitive stimulation reduces IPSP driving force and conductance in the hippocampus in vitro. J Neurophysiol 61:501-511.

Thompson SM, Deisz RA, Prince DA (1988) Relative contributions of passive equilibrium and active transport to the distribution of chloride in mammalian cortical neurons. J Neurophysiol 60:105-124.

Thomson AM (1997) Activity-dependent properties of synaptic transmission at two classes of connections made by rat neocortical pyramidal axons in vitro. J Physiol (Lond) 502:131-147.

Thomson AM, Deuchars J, West DC (1993) Large, deep layer pyramidpyramid single axon EPSPs in slices of rat motor cortex display paired pulse and frequency-dependent depression, mediated presynaptically and self-facilitation, mediated postsynaptically. J Neurophysiol 70:2354-2369.

Thomson AM, West DC, Hahn J, Deuchars J (1996) Single axon IPSPs elicited in pyramidal cells by three classes of interneurones in slices of rat neocortex. J Physiol (Lond) 496:81-102.

Tong G, Shepherd D, Jahr CE (1995) Synaptic desensitization of NMDA receptors by calcineurin. Science 267:1510-1512.

Troyer TW, Miller KD (1997) Physiological gain leads to high ISI variability in a simple model of a cortical regular spiking cell. Neural Comput 9:971-983.

Troyer TW, Krukowski AE, Priebe NJ, Miller KD (1998) Contrastinvarient orientation tuning in cat visual cortex: thalamocortical input tuning and correlation-based intracortical connectivity. J Neurosci 18:5908-5927.

Tsodyks MV, Markram H (1997) The neural code between neocortical pyramidal neurons depends on neurotransmitter release probability. Proc Natl Acad Sci USA 94:719-723.

Tsodyks MV, Sejnowski TJ (1995) Rapid state switching in balanced cortical network models. Network 6:1-14.

van Vreeswijk C, Sompolinsky H (1996) Chaos in neuronal networks with balanced excitatory and inhibitory activity. Science 274:1724-1726.

Varela JA, Richards K, Nelson SB (1995) Modulation of synaptic inputs to visual cortical neurons by adenosine and 5-HT. Soc Neurosci Abstr 21:1653.

Varela JA, Sen K, Gibson J, Fost J, Abbott LF, Nelson SB (1997) A quantitative description of short-term plasticity at excitatory synapses in layer $2 / 3$ of rat primary visual cortex. J Neurosci 17:7926-7940.

Wang LY, Kaczmarek LK (1998) High-frequency firing helps replenish the readily releasable pool of synaptic vesicles. Nature 394:384-388.

Wilcox KS, Dichter MA (1994) Paired-pulse depression in cultured hippocampal neurons is due to a presynaptic mechanism independent of $\mathrm{GABA}_{\mathrm{B}}$ autoreceptor activation. J Neurosci 14:1775-1788. 